\title{
Uso racional de modelos animais para pesquisa e ensino de microcirurgia
}

\section{Rational use of animal models for research and microsurgery training}

\author{
Marcio Rogério Kinshoku'; Carlos Alberto lopez Rodriguez ${ }^{1}$; Ramiro de Souza Fidalgo²; Cinthya Cosme Gutierrez Duran; \\ Pedro Luiz Squilacci Leme, TCBC-SP4; Ivone da Silva Duarte ${ }^{5}$
}

\author{
RE S U M O
}

\begin{abstract}
O objetivo deste estudo é divulgar um modelo experimental de baixo custo, que respeita as normas regulamentadoras das experiências com animais Utilizando animais mortos em outros experimentos contribuindo com a melhora do aprendizado de microcirurgia. São utilizados cadáveres de ratos da raça Wistar, oriundo de descarte de outros cursos. Os cadáveres dos ratos descongelados são utilizados para o treinamento básico de microcirurgia dos acadêmicos do curso de medicina da Universidade Nove de Julho, visando contribuir para o treinamento de acadêmicos, sua utilização se justifica pelo baixo custo do material empregado e muitos animais são poupados durante a fase intermediária do desenvolvimento de habilidades para realização das micro-anastomoses. Este modelo experimental é de baixo custo e permite a utilização racional de animais, corroborando com as normas éticas da pesquisa experimental e contribuindo com o ensino e treinamento da microcirurgia.
\end{abstract}

Descritores: Cirurgia experimental. Wistar. Microcirurgia. Ética. Ensino.

\section{INTRODUÇÃO}

O ensino médico deve ter uma base sólida em todos os níveis de atenção à saúde: primário, secundário e terciário. Uma formação ética digna, com respeito ao paciente, humanização e responsabilidade com a sociedade na qual o futuro médico encontrará seu mercado de trabaIho, quando deverá apresentar competência técnica e teórica atributos fundamentais para o exercício da profissão.

Nas disciplinas cirúrgicas são utilizados diversos métodos de ensino e cursos práticos para se atingir uma meta adequada, complementando a graduação do acadêmico ${ }^{1}$. Devido à grande carga horária do estudante de Medicina, conciliar teoria e prática requer estratégias para a complementação curricular com algumas atividades como estágios, plantões em pronto socorro, acompanhar cirurgiões e participar de projetos de pesquisas. Os acadêmicos do curso de Medicina da Universidade Nove de Julho dispõem de cursos, ligas acadêmicas e participam de projetos de pesquisa para ampliar o seu conhecimento teórico e prático.

O ensino de microcirurgia durante a graduação está sendo difundido em vários centros e para se conseguir este objetivo, o desenvolvimento de modelos experimentais para treinamento tem sido um constante desa- $\mathrm{fio}^{2,3}$. Os modelos experimentais desenvolvidos para o Curso Básico de Microcirurgia devem permitir que monitores treinados tenham condições de ensinar as bases da microcirurgia e cirurgia experimental, instruindo com segurança as normas para o manuseio de animais de pesquisa, do instrumental para microcirurgia e do microscópio cirúrgico ${ }^{4}$. As atividades práticas incluem a utilização de materiais não orgânicos ${ }^{5,6}$, com suturas em materiais sintéticos que simulam o tecido orgânico ${ }^{7}$, suturas em material orgânico conservado pelo frio e depois descongelado, assim como, após o desenvolvimento das aptidões básicas, a utilização de animais vivos, sequência que minimiza o desperdício de animais ${ }^{8}$ nas fases iniciais do aprendizado.

O objetivo deste artigo é divulgar um modelo experimental de baixo custo, que respeita as normas regulamentadoras das experiências com animais. Utilizando animais mortos em outros experimentos de cursos das áreas relacionadas às Ciências da Saúde, que habitualmente não teriam qualquer utilidade, pode ser obtido material orgânico de ótima qualidade para o treinamento dos alunos durante a fase intermediária do programa, etapa preparatória fundamental para o uso de animais vivos, contribuindo com a melhora desta fase do aprendizado de microcirurgia.

Trabalho realizado no Laboratório da Disciplina de Técnica Operatória Experimental do Núcleo Integrado de Simulação (NIS) Dr. Luiz Roberto Barradas Barata - Unidade Vergueiro - UNINOVE. - SP -BR.

1. Acadêmico de Medicina da Universidade Nove de Julho (UNINOVE) - São Paulo - SP-BR; 2. Biólogo da Universidade Nove de Julho (UNINOVE) - São Paulo - SP-BR; 3. Coordenadora dos Laboratórios da Saúde - UNINOVE - São Paulo - SP-BR; 4. Professor da Faculdade de Medicina UNINOVE - São Paulo - SP-BR; 5. Professora Coordenadora do Curso de Medicina - UNINOVE - São Paulo - SP -BR. 


\section{DESCRIÇÃO DO MODELO}

O protocolo de pesquisa foi submetido e autorizado pela Comissão de Ética no Uso de Animais (CEUA) da Universidade Nove de Julho com o parecer de número 0032/2010.

São utilizados cadáveres de ratos da raça Wistar, com $200 \mathrm{~g}$ a $500 \mathrm{~g}$, provenientes do Biotério Central da Universidade Nove de Julho . Os animais mortos são oriundos de experimentos e aulas práticas das áreas de Ciências da Saúde da instituição, e são congelados para serem posteriormente descartados conforme as normas sanitárias vigentes.

Os cadáveres dos ratos descongelados são utilizados para o treinamento básico de microcirurgia dos acadêmicos do curso de medicina da Universidade Nove de Julho, realizado no Laboratório de Técnica Operatória Experimental do Núcleo Integrado de Simulação (NIS) "Dr. Luiz Roberto Barradas Barata". Na segunda fase do programa, quando os alunos, supervisionados por monitores treinados, adquirem habilidades com tecido orgânico antes de operar animais vivos.

Realiza-se a tricotomia da região inguinal, onde se realiza uma incisão oblíqua, ao longo do ligamento, e os vasos femorais são dissecados,. Com auxílio do microscópio cirúrgico realiza-se a dissecção da artéria e da veia femoral, que são cateterizadas com dispositivo flexível de teflon montado sobre agulha números 24 e 22 respectivamente (Figura 1). Após a cateterização se infunde solução de cloreto de sódio a $0.9 \%$ com corante vermeIho para facilitar a visualização dos vasos (Figura 2), utilizado bomba de infusão (Pilot Anestesia, type TCIP, Fresenius Vial ${ }^{\mathrm{TM}}$ ).

Quando os vasos ficam túrgidos ${ }^{9}$ podem ser utilizadas outras opções, como a aorta, ilíacas e carótidas. Visando o treinamento microcirúrgico podem ser feitas anastomoses término-terminais ou término-laterais em qualquer um deles. A dissecção deve permitir o isolamento e clampeamento de um segmento vascular com diâmetro e comprimento adequados, que será seccionado e posteriormente anastomosado com fio de náilon 9-0. A anastomose será realizada com micro suturas de 4 a 8 pontos simples e o microclampe será liberado para que o fluxo seja restabelecido. A perviedade da anastomose será confirmada com testes de patência vascular ou através da visualização pelo microscópio (Figura 3).

\section{DISCUSSÃO}

Em muitos centros especializados o ensino de microcirurgia tem sido realizado por diversos métodos e modelos de ensino, envolvendo ou não animais. No Brasil, estes modelos são utilizados para o aprendizado em áreas como a Cirurgia Plástica, a Microcirurgia Reconstrutiva, a Urologia e a Neurocirurgia² ${ }^{2}$



Figura 1 - Visão com auxílio do microscópio cirúrgico da dissecção da artéria e da veia femoral, que estão cateterizadas com dispositivo flexível de teflon.

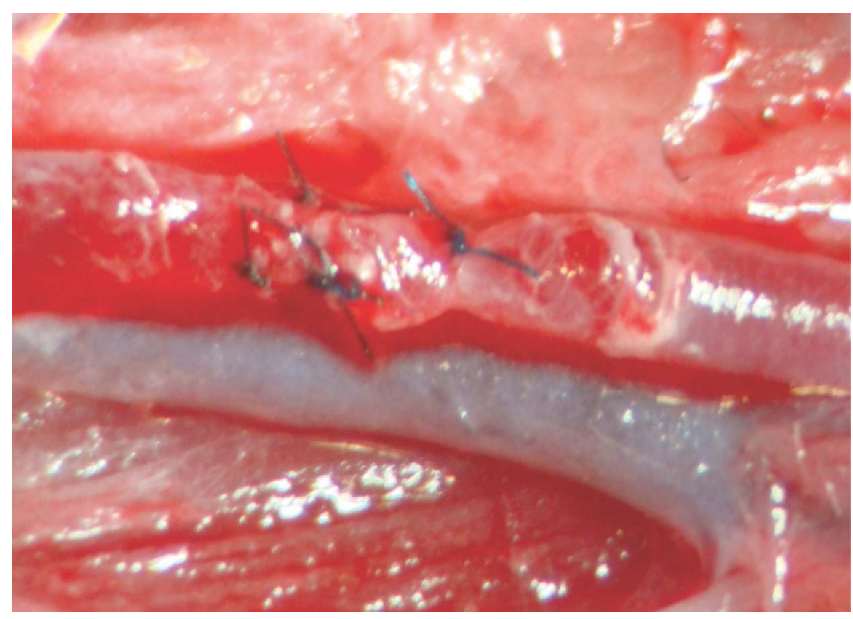

Figura 2 - Visão com auxílio do microscópio cirúrgico em aumento de 2,4x da dissecção da artéria e da veia femoral, Anastomose em $V$ com reparo em sua região distal. Uso de corante foi usado para mimetizar o sangue.



Figura 3 - Visão com auxílio do microscópio cirúrgico em aumento de $1.2 x$ da dissecção da artéria e da veia femoral, Anastomose em $V$ com reparo em sua região distal. Uso de corante foi usado para mimetizar o sangue. 
O treinamento adequado é fundamental, mas exaustivo, exige dedicação, paciência e tempo ${ }^{2,3,7}$. O uso racional de modelos animais em pesquisas biomédicas é uma necessidade até que sejam desenvolvidos modelos e sistemas alternativos que possam se igualar aos modelos animais $^{10}$. Minor et. al. chamam a atenção sobre a meta primordial do estudo experimental e o uso de modelos animais, que auxiliam a solucionar problemas na prática clínica, contribuindo com o desenvolvimento de novos métodos na busca da cura e alívio de doenças ${ }^{11}$. Na área cirúrgica, a prática leva cada vez mais à perfeição, onde a persistência do jovem acadêmico que deseja ser cirurgião faz com que ele tente cada vez mais aprimorar suas habilidades o mais cedo possível, para se adequar ao ambiente cirúrgico.

John Hunter, cirurgião e cientista pioneiro no estudo do assunto, afirmou que o desenvolvimento de novas técnicas cirúrgicas ou habilidades deve ser adquirido em modelos animais com características próximas ao tecido orgânico ${ }^{10}$. Novos modelos são aperfeiçoados nos laboratórios de técnica operatória experimental, onde futuros cirurgiões podem treinar e aperfeiçoar suas habilidades. Em nossa instituição temos a disponibilidade de laboratórios avançados e básicos de técnica operatória, onde futuros cirurgiões podem exercer atividades práticas com monitores acadêmicos sempre auxiliando e iniciando projetos de pesquisa.

Na microcirurgia, o laboratório de treinamento é útil para a aquisição de novas habilidades cirúrgicas ou para o aperfeiçoamento das habilidades já adquiridas, pois funciona de forma adjuvante à sala de cirurgia, reproduzindo situações similares às do ambiente hospitalar ${ }^{12,13}$. Nosso Laboratório de Microcirurgia Experimental dispõe de equipamentos e materiais adequeados para a simulação de ambiente cirúrgico, com todos os rigores da assepsia adequada para que o acadêmico possa se habituar aos rigores do centro cirúrgico.

Muitos modelos foram descritos para o treinamento de habilidades em microcirurgia, incluindo o uso de luvas, placenta, tubos de silicone, folhas, entre outros e continuam importantes nas fases iniciais do treinamento ${ }^{7}$. Hassan et al. ${ }^{14}$ reforçaram o conceito de se promover $\mathrm{o}$ desenvolvimento de meios alternativos aos modelos animais, reduzindo o sofrimento desnecessário. Compreendendo a importância do desenvolvimento de novos modelos, que sejam viáveis e de baixo custo para a instituição, foi desenvolvido este modelo que se utiliza de cadáveres conservados pelo frio, oriundos de outros cursos da Área da Saúde, que são totalmente viáveis pois seu tecido orgânico é preservado e com isso a possibilidade do sistema de preenchimento vascular com solução salina e corantes para dar viscosidade e manter o mais próximo possível do verdadeiro, ou seja, vivo, para que o futuro cirurgião tenha em mente que esta lidando com um ser vivo, e não um animal morto preservado; deste modo o estudante se prepara para seu próximo passo que é a utilização de animais vivos.

Segundo as leis europeias, americanas e canadenses devem ser usados primeiro modelos não animais antes de se realizar o treino cirúrgico em animais vivos ${ }^{15}$, corroborando com nosso modelo que minimiza o desperdício e sofrimento de animais para o importante treino intermediário em microcirurgia.

Os procedimentos alternativos, incluindo laboratórios e workshops devem ser incentivados para familiarizar os estudantes e médicos residentes com o uso adequado dos instrumentos e equipamentos específicos.

Embora o treinamento em laboratório seja satisfatório, recomenda-se que sejam realizadas avaliações periódicas informais para comprovação da evolução do treinamento do individuo ${ }^{2}$..

O modelo de experimental apresentado neste trabalho é de baixo custo e permite a utilização racional de animais, corroborando com as normas éticas da pesquisa experimental e contribuindo com o ensino e treinamento da microcirurgia.

\title{
A $\quad B \quad S \quad T \quad R \quad A \quad C \quad T$
}

\begin{abstract}
The objective of this study is to disclose an experimental model of low cost, which complies with the regulatory norms of animal experiments by using dead animals from other experiments, contributing to the improvement of microsurgery learning. Cadavers of Wistar rats, originated from disposal of other courses, are used. The corpses of thawed mice are used for basic microsurgical training of medical students at the Nove de Julho University, aiming to contribute to the students' training. Their use is justified by the low cost of the material used, many animals being spared during the intermediate phase of skill development for realization of micro-anastomoses. This experimental model is inexpensive and allows the rational use of animals, corroborating with the ethical standards of experimental research and contributing to the education and training of microsurgery.
\end{abstract}

Key words: Models. Microsurgery. Ethics. Hospital. Teaching

\section{REFERENCIAS}

1. Purim K.S.M. Oficina de cirurgia cutânea. Rev Col Bras Cir. 2010;4(37):303-5
2. Jaeger M.R., Silva J. B., Neto N. A., Cristaldo K.A.S. The Rat femoral vessels as training model for digital replantation. Revista da AMRIGS 2009;53(2):128-34.

3. Scholz M, Mucke T, Holzle F, Schmieder K, Engelhardt M, Pechlivanis L, Harders A G. A Program Of Microsurgical Training 
For Young Medical Student: Are Yonger Students Better? microsugery. 2006;26:450-5.

4. Bunckle H.J. The Manual of microvascular surgery : a step-by-step guide to microvascular anastomosisin the rat femoral and vein. unit m, editor. San Francisco: Ralph K. Davis Medical Center; 1975.

5. Lausada N.R, Escudero E, Lamonega R, Dreizzen E, Raimondi JC. Use Of Cryopreserved Rat Arteries for Microsurgical Training. J. Microsugery. 2005;25:500-1

6. Wolff KD, Kesting M, Mucke T, Rau A, Holzle F. Thiel Embalming Technique: A Valuable Method For Microvascular Exercise And Teaching Of Flap Raising microsugery. 2008;23:273-8.

7. Mubin H TZ, Savaci N. . A Non-animal model for microsurgical training with adventitial stripping Plast Reconst Surg. 2000;44:94461.

8. Grober ED, Hamstra SJ, Wanzel KR, Reznick RK, Matsumoto ED, Sidhu RS, and Jarvi KA. The Educational Impact of Bench Model Fidelity on the Acquisition of Technical Skill The Use of Clinically Relevant Outcome Measures. Annals of Surgery. 2004;(240):37481.

9. Blaufox HBLaMD. Blood Volume in the Rat. The Journal of Nuclear Medicine. 1984;25:72-6.

10. Chow PHK. The Rationale for the use os animal models in biomedical research. Editor Chow PKH, Ng RTH, Ogden BE. Using Animal Model in Biomedical Research. $1^{\text {st }}$ edition.Singapure.2008.p.2-10

11. Minor $T$, Klauke $H$, Isselhard W. Resuscitation of cadaveric livers from non-heart-beating donors after warm ischemic insult: a novel technique tested in the rat. Experientia. 1996 Jul 15;52(7):661-4.
12. Klein I SU, Timmermann W, Thiede A, Gassel HJ. Microsurgical training course for clinicians and scientists at a German University Hospital: 10 years experience microsurgery. 2003;5:461-5.

13. Wanzel KR ME, Stanley JH, Dimitri JA,. Teaching technical skills: training on a Simple, Inexpensive, and Portable model. Plast Reconst Surg. 2002;1:258-63.

14. A. Z. Hassan KBK, BD Remi-adwumi, C.A. Awasum and M. T. Abubakar. Animal models in surgical training: Choise and Ethics. Nigerian Journal of Surgical research. 2005:17:260-7.

Recebido em 10/06/2011

Aceito para publicação em 15/08/2011

Conflito de interesse: nenhum

Fonte de financiamento: nenhuma

\section{Como citar este artigo:}

Kinshoku MR, Rodriguez CAL, Fidalgo RS, Duran CCG, Leme PLS, Duarte IS. Uso sustentável de modelos animais para pesquisa e ensino de microcirurgia. Rev Col Bras Cir. [periódico na Internet] 2012; 39(5). Disponível em URL: http://www.scielo.br/rcbc

\section{Endereço para correspondência:}

Marcio Rogério Kinshoku

E-mail: mrkinshoku@uol.com.br 